\title{
129. Evidence for Rotation of Dye Molecules in Membrane Macromolecules Associated with Nerve Excitation
}

\author{
By Akira WARASHINA**) and Ichiji TASAKI*) \\ (Comm. by Yasuji KATsuki, M. J. A., Sept. 12, 1975)
}

The nerve membrane where the electrophysiological excitation process takes place is known to be composed of macromolecules with a highly ordered structure. The existence of such an ordered structure has been revealed by incorporating certain types of fluorescent probes into the membrane and subsequently measuring changes in emission of highly polarized fluorescence. ${ }^{1)}$ The present communication describes the results of an experiment indicating again that dye molecules are more-or-less regularly aligned in the nerve membrane. In addition, experimental evidence is presented showing that the geometric axes of elongated dye molecules in the membrane change when an action potential is produced in the nerve.

Experimental. Crab nerves stained with a variety of dyes were used. The method of staining nerves and recording optical responses was the same as that used in the experiment described in the preceding communication, ${ }^{2)}$ except that the monochromatic light used for measurements was polarized by insertion of a Polaroid HN 32 sheet in the lightpath. Small changes in the intensity of the light transmitted through the stained nerve were recorded with a photodiode (see Fig. 1, left). To improve the signal-to-noise ratio, the optical responses were averaged over 32 trials. Measurements of the optical responses involve detection of small changes in the light intensity in the presence of a strong background light. In the present series of measurements, the intensity of the background light was adjusted so that the D.C. output of the detector was constant irrespective of the wavelength. The amplitude of the recorded response was therefore proportional to the change in the absorbance of the dye associated with action potentials at each wavelength. All the measurements were carried out at room temperature $\left(18-20^{\circ} \mathrm{C}\right)$.

It was found that the wavelength-dependence of the optical response is strongly influenced by the polarization of the light used for measurements. Fig. 1 shows the results obtained by this procedure using crab nerves stained with Eastman merocyanine-540

\footnotetext{
*) Laboratory of Neurobiology National Institute of Mental Health Bethesda, Maryland, U. S. A.

**) Kanazawa Medical University, Ishikawa Prefecture, Japan.
} 

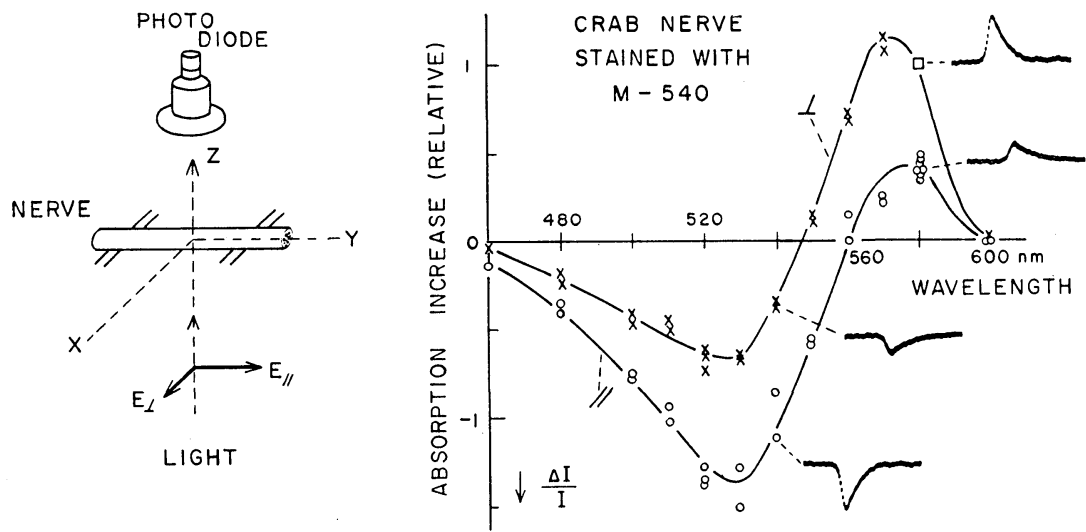

Fig. 1. Spectra of absorption changes associated with action potentials obtained from crab nerves stained with merocyanine-540. Circles indicate the data obtained with light polarized in the parallel direction $\left(E_{\|}\right)$and crosses indicate those obtained under perpendicular polarization $\left(E_{\perp}\right)$. $580 \mathrm{~nm}$ was chosen as reference wavelength. Four examples of computer records, from which the spectra were constructed, are shown.
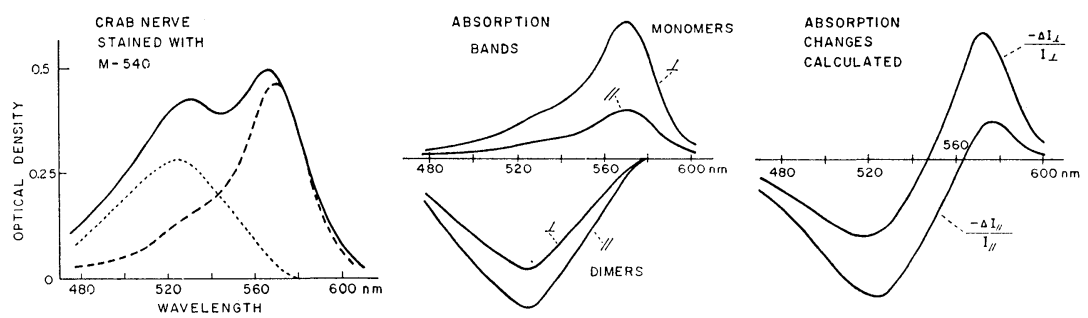

Fig. 2. Reconstruction of spectra of absorption changes during nerve excitation (see text).

(abbreviated as M-540), a dye shown by Davila et al.3) to be a powerful membrane probe. M-540 is an elongated molecule with two heterocyclic nuclei connected by conjugated double-bonds composed of 4 carbon atoms. It is seen in the figure that the spectrum of the optical response observed with the light polarized with its electric vector parallel to the nerve is very different from the spectrum obtained with light polarized in the perpendicular direction. It is to be noted that the wavelength at which the sign of the response reverses is very different between the two spectra. The dependence of the reversal point on the wavelength was not limited to merocyanine; it was observed also with methyl violet, crystal violet, etc.

Discussion of Results. The experimental result shown in Fig. 1 is now interpreted on the basis of the spectroscopic characteristics of the dye. By mounting a stained crab nerve in a special chamber, the absorption spectrum of M-540 in a crab nerve has been deter- 
mined with a Beckman spectrophotometer. ${ }^{4)}$ The solid line in Fig. 2, left, shows the result of those previous determinations. In this absorption spectrum, there are two maxima, one at $528 \mathrm{~nm}$ and the other at about $565 \mathrm{~nm}$. The dashed line in this figure represents the corrected fluorescence excitation spectrum of the dye in a crab nerve. This spectrum has a single maximum at about $568 \mathrm{~nm}$. If the light energy absorbed by a single species of dye molecules is converted into fluorescent light by a simple process of fluorescence emission, the fluorescence excitation spectrum is expected to coincide with the absorption spectrum (except for the dimension of the ordinate). The existence of an additional maximum in the absorption spectrum indicates that there is a non-fluorescent species of dye molecules which have a different absorption spectrum. Based on the effect of dilution of the dye in chloroform, the absorption band with a maximum of about $528 \mathrm{~nm}$ is a attributed to dimers of the dye molecules. The dotted line in Fig. 2, left, represents the absorption spectrum of the dimers obtained by subtraction of the monomer spectrum (shown by the dashed line) from the total absorption band (the solid line). It has been proposed that in the nerve membrane stained with M-540 optical responses are produced by conversion of dimers into monomers during action potentials. ${ }^{3)}$ The middle and right diagrams show that the observed spectrum of the optical responses can adequately be interpreted as being nothing but linear combinations of the monomer and dimer spectra shown in Fig. 2, left. The absorption spectra shown in the right-hand diagram are constructed by subtracting the dimer absorption bands from the monomer absorption bands in the middle diagram. The absorption bands in the middle are obtained from the monomer and dimer bands shown in the left-hand diagram [by multiplying proper factors]. Since the spectrum of the optical response (shown in Fig. 1) is strongly affected by the polarization of the lightwave used for measurements, two sets of absorption spectra are required to construct the observed changes in absorbance associated with action potentials. It is seen in the figure that the spectra obtained by this calculation reproduce all the essential characteristics of the observed spectra of the optical responses.

It is important to note that the ratio between the two monomer band amplitudes in the middle diagram is very different from that between the two dimer band amplitudes in the same diagram. The monomer absorbance observed with perpendicularly polarized lightwave is shown to be far stronger than that seen with lightwave polarized in the parallel direction. In contrast, the two dimer absorption bands in the figure are comparable in amplitude. This finding 

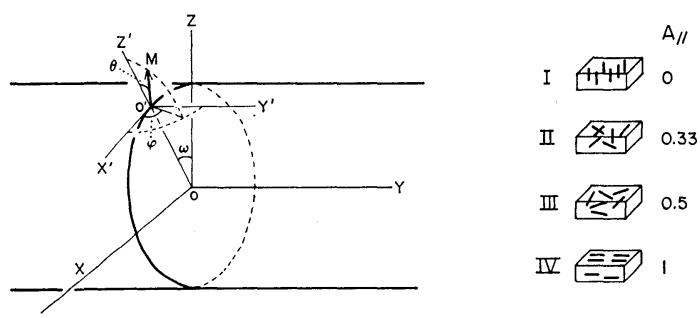

Fig. 3. Diagram illustrating three angles, $\theta, \varphi$ and $\omega$, used in theoretical consideration of the distribution of transition moments of dye molecules in the nerve membrane. Axis $O Y$ represents the long axis of a single fiber in the nerve trunk (see text).

may be explained in the following manner: When dimers with their molecular axes directed parallel to the membrane surface are converted into monomers during action potentials, the orientation of the dye molecules changes. As a result of such rotation of dye molecules, the ratio between the number of molecules with different spatial orientation changes when the nerve membrane is in its electrophysiologically excited state.

Mathematical treatment of two discrete states of dye molecules in nerve membrane. The probability of absorption of light by a dye molecule is proportional to the square of the cosine of the angle between the electric vector of the incident lightwave and the direction of the absorption oscillator (or the transition moment) of the dye molecules. Fig. 3 illustrates the spatial relationship between the nerve membrane and a dye molecule bound to the membrane. Axis $O Y$ represents the longitudinal axis of a particular single fiber in the nerve trunk under study. As shown in Fig. 1, left, the incident light is considered to propagate in the direction of axis $O Z$ and is polarized with its electric vector directed along either axis $O Y$ (parallel polarization) or axis $O X$ (perpendicular polarization). The angle which the axis of the oscillator $\left(O^{\prime} M\right)$ makes with the normal $\left(O^{\prime} Z^{\prime}\right)$ to the membrane surface is denoted by $\theta$. As illustrated in the figure, two additional angles, $\varphi$ and $\omega$, are required to denote the orientation of $O^{\prime} M$ relative to the electric vector of the incident light. The cosine of the angle between $O^{\prime} M$ and $O^{\prime} Y^{\prime}$ is given by $[\sin \theta \cdot \sin \varphi$ ].

The angular distribution of the dye moles relative to the membrane surface may be denoted by a function of $\theta$ and $\varphi, f(\theta, \varphi)$. Function $f(\theta, \varphi)$ is normalized, namely,

$$
\int_{0}^{2 \pi} \int_{0}^{\pi} f(\theta, \varphi) \sin \theta d \theta d \varphi=1
$$

so that $[f(\theta, \varphi) \sin \theta d \theta d \varphi]$ represents the probability of finding a dye molecule with its orientation in the range between $\theta$ and $(\theta+d \theta)$ and 
$\varphi$ and $(\varphi+d \varphi)$.

The molar extinction coefficient of the dye in the membrane is now denoted by $\varepsilon(\lambda)$. Then, the contribution of these dye molecules to absorption of light polarized along $O Y$ is proportional to the product $\varepsilon(\lambda) A_{\|}$where $A_{\|}$, a geometric factor representing the "degree of parallel orientation" of the dye molecules, is defined by

$$
A_{\|}=\iint f(\theta, \varphi)[\sin \theta \sin \varphi]^{2} \sin \theta d \theta d \varphi
$$

The quantity in the bracket represents the cosine of the angle between $O^{\prime} M$ and $O^{\prime} Y^{\prime}$. Again, the integration extends from 0 to $\pi$ for $\theta$ and from 0 to $2 \pi$ for $\varphi$. When the axis of every dye molecule in the membrane is oriented perpendicularly to the surface (case I in the figure), $A_{\|}=0$. For a completely random spatial distribution (Case II), namely for $f(\theta, \varphi)=1 /(4 \pi), A_{\|}=1 / 3$. When the dye molecules are randomly distributed with their axes parallel to the membrane surface (case III), $A_{\|}=0.5$. When they are all parallel to the long axis of the nerve (case IV), $A_{\|}=1$. In an analogous manner, the geometric factor reflecting the degree of perpendicular orientation of the dye molecules, $A_{\perp}$, is defined by

$$
A_{\perp}=\frac{1}{2 \pi} \iiint f(\theta, \varphi)[\cos \theta \cdot \sin \omega+\sin \theta \cdot \cos \varphi \cdot \cos \omega]^{2} \sin \theta d \theta d \varphi d \omega
$$

the limits of integration with respect to $\omega$ being from 0 to $2 \pi$. By comparing this equation with equations 1 and 2 , it is easily found that $A_{\|}$and $A_{\perp}$ are related to each other by the relation

$$
A_{\|}+2 A_{\perp}=1
$$

Under the experimental conditions illustrated in Fig. 1, there are many nerve fibers in the light path. The contribution of the dye molecules in the membrane to the optical density measured under the conditions of parallel and perpendicular polarization is equal to $3 N \varepsilon(\lambda) A_{\|}$and $3 N \varepsilon(\lambda) A_{\perp}$, respectively, where $N$ represents the average dye concentration times the thickness of the nerve. The factor of 3 is required because when the dye distribution is completely random, namely when $A_{\|}=A_{\perp}=1 / 3$, the optical density must be given by $N \varepsilon(\lambda)$ irrespective of the polarization of the incident light.

The molar extinction coefficients of monomers and dimers are denoted by $\varepsilon_{1}(\lambda) / 3$ and $\varepsilon_{2}(\lambda) / 3$, respectively. In general, the spatial distribution of the dimers in the membrane must be different from that of monomers. By substituting $f(\theta, \varphi)$ in equations 2 and 3 for $f^{\prime}(\theta, \varphi)$, the distribution function for dimer molecules, the geometric factors for the dimer species $B_{\|}$and $B_{\perp}$, are defined: $B_{\|}+2 B_{\perp}=1$. Then, the intensities of the light transmitted through a stained nerve are given by

$$
I_{\|}=I_{0} \cdot K \cdot \exp \left\{-N_{1} A_{\| \varepsilon_{1}}(\lambda)-N_{2} B_{\|} \varepsilon_{2}(\lambda)\right\}
$$


under parallel polarization, and

$$
I_{\perp}=I_{0} \cdot K \cdot \exp \left\{-N_{1} A_{\perp} \varepsilon_{1}(\lambda)-N_{2} B_{\perp} \varepsilon_{2}(\lambda)\right\}
$$

under perpendicular polarization. Here, the intensity of the incident light is denoted by $I_{0}$, and the reduction of light intensity due to the turbidity of the nerve and to the dye molecules bound to non-responsive sites (connective tissue, Schwann's cells, etc.) by factor $K$, and the amount of monomers and dimers in the lightpath by $N_{1}$ and $N_{2}$, respectively. [Note that the dye molecules bound to non-responsive sites are randomly oriented.]

Finally, changes in the light intensity associated with conversion of a small portion of dimers into monomers are discussed. On account of mass conservation, this conversion proceeds under the condition that $\left(N_{1}+2 N_{2}\right)=$ constant, or $\Delta N_{1}=-2 \Delta N_{2}$ for small changes. Therefore, the changes in the light intensity, $\Delta I_{\|}$and $\Delta I_{\perp}$, produced by such conversion, are given by

and

$$
-\frac{\Delta I_{\|}}{I_{\|}}=\left[A_{\|} \varepsilon_{1}(\lambda)-B_{\|} \frac{1}{2} \varepsilon_{2}(\lambda)\right] \Delta N_{1}
$$

$$
-\frac{\Delta I_{\perp}}{I_{\perp}}=\left[A_{\perp} \varepsilon_{1}(\lambda)-B_{\perp} \frac{1}{2} \varepsilon_{2}(\lambda)\right] \Delta N_{1}
$$

Equations 7 and 8 constitute the theoretical basis for our interpretation of the changes in light absorption observed in M-540 stained crab nerves. It is clear from the explanation of Fig. 2 that the four curves in the middle diagram are represented by the four terms on the right-hand side of equations 7 and 8 . From the ratio between the amplitudes of the two monomer bands in the diagram it is found that $A_{\perp}: A_{\|}$is roughly 2.8 , indicating that $A_{\|}$is approximately 0.15. Similarly, from the relative amplitudes of the two dimer bands in the diagram, the ratio of $B_{\perp}$ to $B_{\|}$is found to be 0.74 . From this it follows that $B_{\|}$is roughly 0.40 . Thus, it was found that the degree of parallel orientation of dye molecules in the excited state of the nerve membrane is very different from that in the resting state.

\section{References}

1) Tasaki, I., Sisco, K., and Warashina, A. (1974): Biophys. Chem., 2, 316.

2) Tasaki, I., and Warashina, A. (1975) : Proc. Japan Acad., 51, 604-609 (1975).

3) Davila, H. V., Salzberg, B. M., and Cohen, L. B. (1973): Nature, New Biology, 241, 159.

4) Tasaki, I., Warashina, A., and Pant, H. (1975): in preparation. 